\title{
Superior Effects of High-Intensity Interval Training Compared to Conventional Therapy on Cardiovascular and Psychological Aspects in Myocardial Infarction
}

\author{
Ha-Yoon Choi, MD, Hee-Jun Han, MD, Ji-won Choi, PT, Han-Young Jung, MD, Kyung-Lim Joa, MD
}

Department of Physical and Rehabilitation Medicine, Inha University School of Medicine, Incheon, Korea

\begin{abstract}
Objective To evaluate the effect of high-intensity interval training (HIIT) on psychological symptoms, activity states, and cardiovascular functions in patients with myocardial infarction (MI) of low and moderate risk stratification.

Methods This prospective study randomly allocated 44 patients with MI to 18 sessions of HIIT or conventional moderate-intensity continuous training (MICT). Outcome measures were assessed at baseline and after 18 sessions.

Results Post-exercise cardiovascular and functional states, maximal oxygen uptake $\left(\mathrm{VO}_{2 \max }\right)$, metabolic equivalents (METs), 6-Minute Walking Test (6MWT), and Korean Activity Scale/Index (KASI) scores were significantly improved in the HIIT group compared to those in the MICT group after 18 exercise sessions. In particular, $\mathrm{VO}_{2 \max }$ was significantly $(\mathrm{p}<0.005)$ improved in the HIIT group $(7.58 \mathrm{~mL} / \mathrm{kg} / \mathrm{min})$ compared to that in the MICT group $(2.42 \mathrm{~mL} / \mathrm{kg} / \mathrm{min})$. In addition, post-exercise psychological states (i.e., scores of Fatigue Severity Scale [FSS] and depression items of the Hospital Anxiety and Depression Scale [HADS_D]) were significantly improved in the HIIT group compared to those in the MICT group after 18 exercise sessions. HADS-D was improved by 1.89 in the HIIT group compared to decrement of 0.47 in the MICT group. FSS was improved by 6.38 in the HIIT group compared to decrement of 0.77 in the MICT group $(\mathrm{p}<0.005)$.

Conclusion This study demonstrates that HIIT can improve cardiac function, psychological, and activity states in low and moderate risk MI patients. Compared to conventional MICT, HIIT can improve cardiovascular functions, activity states, depression, and fatigue more effectively.
\end{abstract}

Keywords Myocardial infarction, Cardiac rehabilitation, High-intensity interval training, Maximal oxygen consumption, Depression

Department of Physical and Rehabilitation Medicine, Inha University School of Medicine, 27 Inhang-ro, Jung-gu, Incheon 22332, Korea. Tel: +82-32890-2486, Fax: +82-32-890-2486, E-mail: drjoakl@gmail.com

ORCID: Ha-Yoon Choi (http://orcid.org/0000-0002-0084-1470); Hee-Jun Han (http://orcid.org/0000-0003-4326-1617); Ji-won Choi (http://orcid. org/0000-0001-9077-8825); Han-Young Jung (http://orcid.org/0000-0003-1428-9821); Kyung-Lim Joa (http://orcid.org/0000-0002-3747-9831).

() This is an open-access article distributed under the terms of the Creative Commons Attribution Non-Commercial License (http://creativecommons.org/ licenses/by-nc/4.0) which permits unrestricted noncommercial use, distribution, and reproduction in any medium, provided the original work is properly cited. Copyright $\odot 2018$ by Korean Academy of Rehabilitation Medicine 


\section{INTRODUCTION}

Coronary heart disease (CHD) accounts for one-third of all deaths globally, with a total of 7.4 million deaths due to CHD in 2013 [1]. Cardiac rehabilitation (CR) is an accepted treatment modality following myocardial infarction (MI) [2]. Exercise-based CR is known to result in a significantly lower risk of fatal and non-fatal re-infarction by improving cardiac and coronary vascular functions as well as CHD risk factor profiles [3]. Current guidelines encourage moderate to high-intensity aerobic exercise for 20-30 minutes for CR, preferably on all days of the week [4].

High-intensity interval training (HIIT) involving repeated 30- to 300-second bouts of aerobic exercise at intensities ranging from $85 \%$ to $100 \%$ of maximal oxygen uptake $\left(\mathrm{VO}_{2 \max }\right)$ interspersed by recovery periods of equal or shorter duration is another form of exercise. The potential of HIIT for CR has been recently investigated [5]. However, the majority of previous studies did not start HIIT immediately in acute phase. The periods from MI to the onset of phase II CR used in previous studies were 3 to 6 months after MI. Furthermore, most studies only enrolled low risk patients or they did not suggest risk stratification for cardiac rehabilitation [5-9]. Therefore, the effect of HIIT on cardiovascular variables, including
$\mathrm{VO}_{2 \max }$, needs to be evaluated in acute phase II patients with defined risk stratification.

High prevalence rates of psychological symptoms and disorders have been reported in patients with MI. These symptoms and disorders have negative impacts on prognosis of patients [10-13]. Apart from improving physical capacity, effects of CR include modification of sympathovagal balance and improved mental competence. Depression and anxiety are commonly observed risk factors in patients after MI. They are also strong predictors of mortality [10-13]. Participation in CR can alleviate manifestations of depression and anxiety [14-17]. However, little is known about the effect of HIIT on psychological symptoms post-MI. Because CR programs are crucial sources for CHD secondary prevention, it is important to determine the optimal training method to improve cardiovascular and psychological symptoms.

Therefore, the aim of this study was to compare effects of HIIT and moderate-intensity continuous training (MICT) CR on cardiovascular functions and psychological symptoms of phase II MI patients with defined risk stratification (low and moderate risk).

\section{MATERIALS AND METHODS}

This study was a prospective, non-blinded, randomized

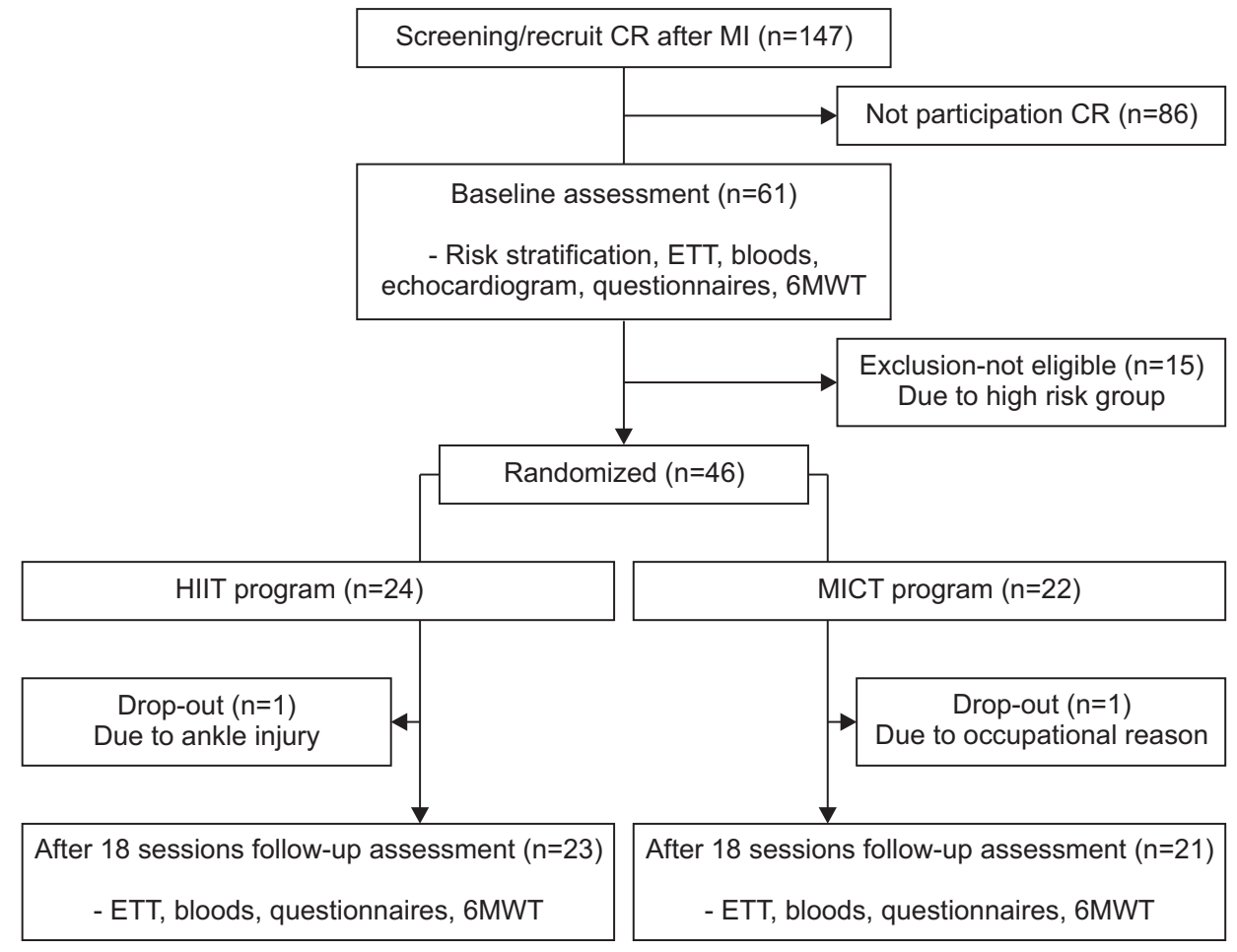

Fig. 1. Study flow chart. CR, cardiac rehabilitation; MI, myocardial infarction; ETT, exercise tolerance test; 6MWT, 6-Minute Walking Test; HIIT, high-intensity interval training; MICT, moderate-intensity continuous training. 
controlled trial. Participants were randomly allocated to 18 sessions of HIIT or MICT training. Outcomes were measured at baseline and after 18 sessions. An outline of the participant pathway for this study is presented in Fig. 1.

\section{Patients}

A total of 44 study subjects had first time ST-segment elevation myocardial infarction (STEMI). They were treated by primary percutaneous coronary intervention (PCI) between October 2015 and March 2017. Risk stratification (low, moderate, and high risk) was performed based on the American Association of Cardiovascular and Pulmonary Rehabilitation. Enrolled patients were in 'low or moderate' group who agreed to receive CR by visiting a rehabilitation clinic within 1-2 weeks after discharge from cardiology department.

A total of 147 patients were admitted to the outpatient department of rehabilitation after MI, of which 86 did not participate in phase II CR program. A total of 61 patients were enrolled for baseline assessment, 15 of which were evaluated to belong to the high-risk group. Therefore, they were excluded from this study. Exclusion criteria included prior PCI or CABG, left ventricular ejection fraction (LVEF) less than $40 \%$, uncontrolled arrhythmia, hypertension, diabetes, or additional issues that would preclude potential participation in the program.

All patients underwent an initial baseline exercise tolerance test (ETT). A total of 24 patients were assigned to the HIIT group while 22 patients were assigned to the conventional MICT group. Two patients (one patient in each group) could not finish all 18 sessions because of ankle injury and occupational reasons. Therefore, 23 patients in the HIIT group and 21 patients in the control group were analyzed in this study. In addition, HIIT and control groups were subdivided into low (7 patients in each group) and moderate (16 in HIIT and 14 in the control) subgroups (Fig. 1).

The number of patients recruited was small, although it was enough to meet the calculated required sample size of 20 patients per group determined by using $\mathrm{G}^{*}$ Power program with a Cohen d effective size of 0.2 , a statistical power of 0.95 , and an error probability of 0.05 .

\section{Interventions}

Patients in the HIIT group warmed up for about $10 \mathrm{~min}$ utes at an intensity of $40 \%-50 \%$ of HR max. HIIT consisting of $4 \times 4$ minute exercise periods at $85 \%-100 \%$ of heart rate maximum $\left(\mathrm{HR}_{\max }\right)$ per session. Exercise periods were separated by a recovery period for 3 minutes at 50\%-60\% of $\mathrm{HR}_{\max }$. Training sessions finished with a cool-down period at $40 \%-50 \%$ of $\mathrm{HR}_{\text {max }}$ [18]. One session consisted of 10 minutes of stretching, 5 minutes of warm-up, $28 \mathrm{~min}$ utes of main exercise, and 5 minutes of cool-down for a total of 48 minutes (Fig. 2).

Members in the control group participated in conventional MICT. For MICT, the intensity was simply the one that the participant could carry on for a prolonged time-period. The subject should be able to converse in full sentences. During MICT sessions, patients worked continuously at an intensity of $60 \%-70 \%$ of $\mathrm{HR}_{\max }$. In the MICT group, exercise program was continued for the same time-period. During 28 minutes of main exercise, the exercise intensity was $60 \%-70 \%$ of $\mathrm{HR}_{\max }$. There were 18 sessions for all patients in each group [18]. Both

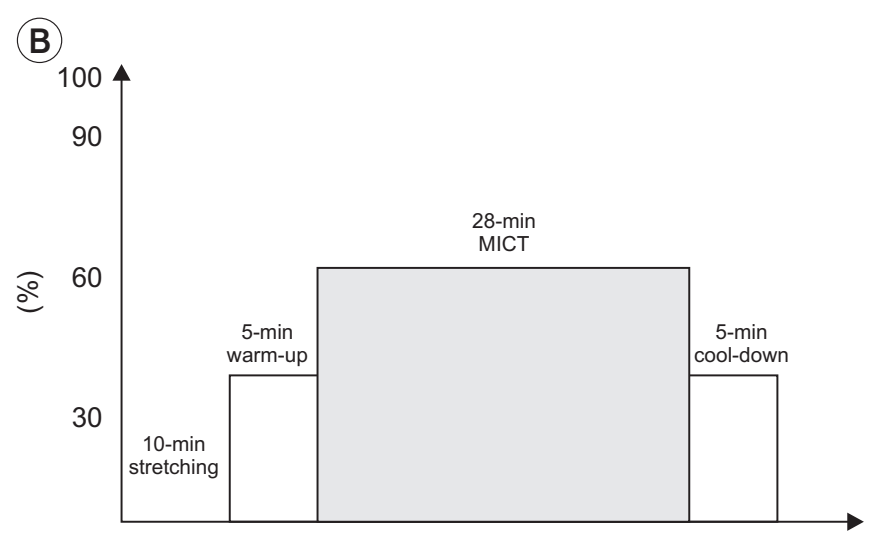

Fig. 2. Comparison of high-intensity interval training (HIIT) and moderate-intensity continuous training (MICT) protocols: (A) HIIT group, (B) MICT group. 
groups were conducted an average of 1-2 sessions per week for 9-10 weeks.

All patients provided written informed consent before study enrollment. This study was approved by the Institutional Review Board of Inha University Medical School (IRB No. 201701025).

\section{Outcome measures}

Cardiovascular function, psychological state, and activity state were evaluated before and after the 18-session exercise program. For cardiovascular functions, the following parameters were evaluated: $\mathrm{LVEF}, \mathrm{VO}_{2 \max }$, metabolic equivalents (METs), $\mathrm{HR}_{\max }$, the 6 -Minute Walking Test (6MWT), maximal systolic blood pressure, and maximal diastolic blood pressure. Korean Activity Scale/Index (KASI) scores were used to determine changes in activity states due to cardiovascular functional improvements.

Psychological states were evaluated using self-administered questionnaires, including the Hospital Anxiety and Depression Scale (HADS) for anxiety and depression, the Patient Health Questionnaire (PHQ-9) for depression, Insomnia Severity Index (ISI) for insomnia, and Fatigue Severity Scale (FSS) for fatigue.

Activity states are one of the most important variables in clinical practice or research. They represent the patient ability, overall condition, and severity of illness [19]. The Duke Activity Status Index (DASI) is a tool that can measure activity capacity [20]. KASI, a Korean version of DASI [19], was used in this study.

HADS, a tool most commonly used to screen levels of anxiety and depression in people with physical problems, was used in this study. Seven of its items are related to anxiety while the other seven items are related to depression [21]. PHQ-9 is a tool for screening, diagnosing, monitoring, and measuring severity of depression. It can be applied repeatedly to access the alleviation or worsening of depression symptoms in response to treatment [22]. FSS is a one of the most commonly used 9-item selfreport questionnaires to assess fatigue in individuals with physical problems [23]. ISI is a 7-item multi-dimensional screening tool for insomnia. It assesses severity of sleep onset and maintenance difficulties, satisfaction with current sleep pattern, interference with daily functioning, and degree of distress or concern caused by the sleep problems [24]. These were used in this study.

\section{Statistics}

The Mann-Whitney test was used to compare group demographic and clinical data, including $\mathrm{VO}_{2 \max }, 6 \mathrm{MWT}$, KASI, HADS, PHQ-9, ISI, and FSS before and after treatment. Repeated measures analysis of variance was used to compare variables before and after intervention between the both groups (time-group interaction). All statistical analyses were performed using SPSS version 18 for Windows (SPSS Inc., Chicago, IL, USA). Statistical significance was accepted at $\mathrm{p}$-value $<0.05$.

\section{RESULTS}

Demographics and clinical characteristics are summarized in Table 1. Mean age of the 44 study subjects was $54.93 \pm 9.91$ years. Of these subjects, $89 \%$ were males. Mean ejection fraction was $46.90 \% \pm 7.14 \%$. Mean BMI was $25.39 \pm 3.06 \mathrm{~kg} / \mathrm{m}^{2}$. Initial mean $\mathrm{VO}_{2 \max }$ was $30.17 \pm 7.56$ $\mathrm{mL} / \mathrm{kg} / \mathrm{min}$. Initial METs was $8.62 \pm 2.16$. $6 \mathrm{MWT}$ was $502.88 \pm 75.99 \mathrm{~m}$. Mean duration from a diagnosis of MI to first ETT was $14.29 \pm 6.53$ days. No demographic or clinical value was significantly different between the HIIT and control MICT groups. Mean durations from diagnosis were

Table 1. Demographics and initial clinical characteristics of subjects

\begin{tabular}{|cccc}
\hline & $\begin{array}{c}\text { Control } \\
(\mathbf{n = 2 1})\end{array}$ & $\begin{array}{c}\text { HIIT } \\
(\mathbf{n = 2 3})\end{array}$ & p-value \\
\hline Age (yr) & $57.31 \pm 12.62$ & $53.00 \pm 6.84$ & 0.251 \\
\hline Sex (male:female) & & & 0.884 \\
\hline Male & 18 & 21 & \\
\hline Female & 3 & 2 & \\
\hline LVEF (\%) & $47.31 \pm 9.63$ & $46.56 \pm 4.54$ & 0.786 \\
\hline Risk stratification & & & 0.202 \\
\hline Low & 7 & 7 & \\
\hline Moderate & 14 & 16 & \\
\hline Number of involved vessels & & 0.110 \\
\hline 1 & 10 & 15 & \\
\hline 2 & 8 & 6 & \\
\hline 3 & 3 & 2 & \\
\hline $\mathrm{BMI}^{\left(\mathrm{kg} / \mathrm{m}^{2}\right)}$ & $26.30 \pm 2.47$ & $24.65 \pm 3.36$ & 0.151 \\
$\mathrm{VO}_{2 \max }(\mathrm{mL} / \mathrm{kg} / \mathrm{min})$ & $27.98 \pm 8.63$ & $31.95 \pm 6.29$ & 0.163 \\
\hline
\end{tabular}

Values are presented as mean \pm standard deviation. HIIT, high-intensity interval training; LVEF, left ventricle ejection fraction; $\mathrm{BMI}$, body mass index; $\mathrm{VO}_{2 \max }$, maximal oxygen consumption. 
Table 2. Pre- and post-training cardiac functions, psychological statuses, and activity states in the two study groups

\begin{tabular}{|c|c|c|c|c|c|}
\hline & \multicolumn{2}{|c|}{ Control } & \multicolumn{2}{|c|}{ HIIT } & \multirow{2}{*}{ p-value } \\
\hline & Pre & Post & Pre & Post & \\
\hline $\mathrm{VO}_{2 \max }(\mathrm{mL} / \mathrm{kg} / \mathrm{min})$ & $27.98 \pm 8.63$ & $30.40 \pm 8.39$ & $31.95 \pm 6.29$ & $39.53 \pm 6.71$ & $0.001^{*}$ \\
\hline METs & $7.99 \pm 2.46$ & $8.81 \pm 2.42$ & $9.13 \pm 1.80$ & $11.30 \pm 1.93$ & $0.004^{*}$ \\
\hline $6 \mathrm{MWT}(\mathrm{m})$ & $505.92 \pm 68.16$ & $561.62 \pm 73.92$ & $500.41 \pm 83.95$ & $606.56 \pm 72.70$ & $0.034^{*}$ \\
\hline KASI & $51.46 \pm 21.84$ & $53.04 \pm 20.14$ & $59.04 \pm 13.36$ & $71.48 \pm 8.05$ & $0.019 *$ \\
\hline HADS_A & $7.05 \pm 3.13$ & $4.76 \pm 3.13$ & $5.63 \pm 3.04$ & $3.94 \pm 2.85$ & 0.449 \\
\hline HADS_D & $5.41 \pm 3.29$ & $5.88 \pm 3.67$ & $6.57 \pm 2.24$ & $4.68 \pm 2.81$ & $0.025^{*}$ \\
\hline PHQ-9 & $4.69 \pm 4.32$ & $3.77 \pm 3.70$ & $5.50 \pm 3.95$ & $2.00 \pm 2.00$ & 0.068 \\
\hline ISI & $5.77 \pm 3.78$ & $5.62 \pm 3.68$ & $7.31 \pm 5.77$ & $4.88 \pm 5.39$ & 0.150 \\
\hline FSS & $23.69 \pm 11.65$ & $24.46 \pm 13.02$ & $27.44 \pm 12.54$ & $19.06 \pm 9.10$ & $0.034^{*}$ \\
\hline
\end{tabular}

Values are presented as mean \pm standard deviation.

HIIT, high-intensity interval training; $\mathrm{VO}_{2 \max }$, maximal oxygen consumption; METs, metabolic equivalents; 6MWT, 6-Minute Walking Test; KASI, Korean Activity Scale/Index; HADS_A, anxiety items of the Hospital Anxiety and Depression Scale; HADS_D, depression items of the Hospital Anxiety and Depression Scale; PHQ-9, Patient Health Questionnaire; ISI, Insomnia Severity Index; FSS, Fatigue Severity Scale.

${ }^{*} \mathrm{p}<0.05$.
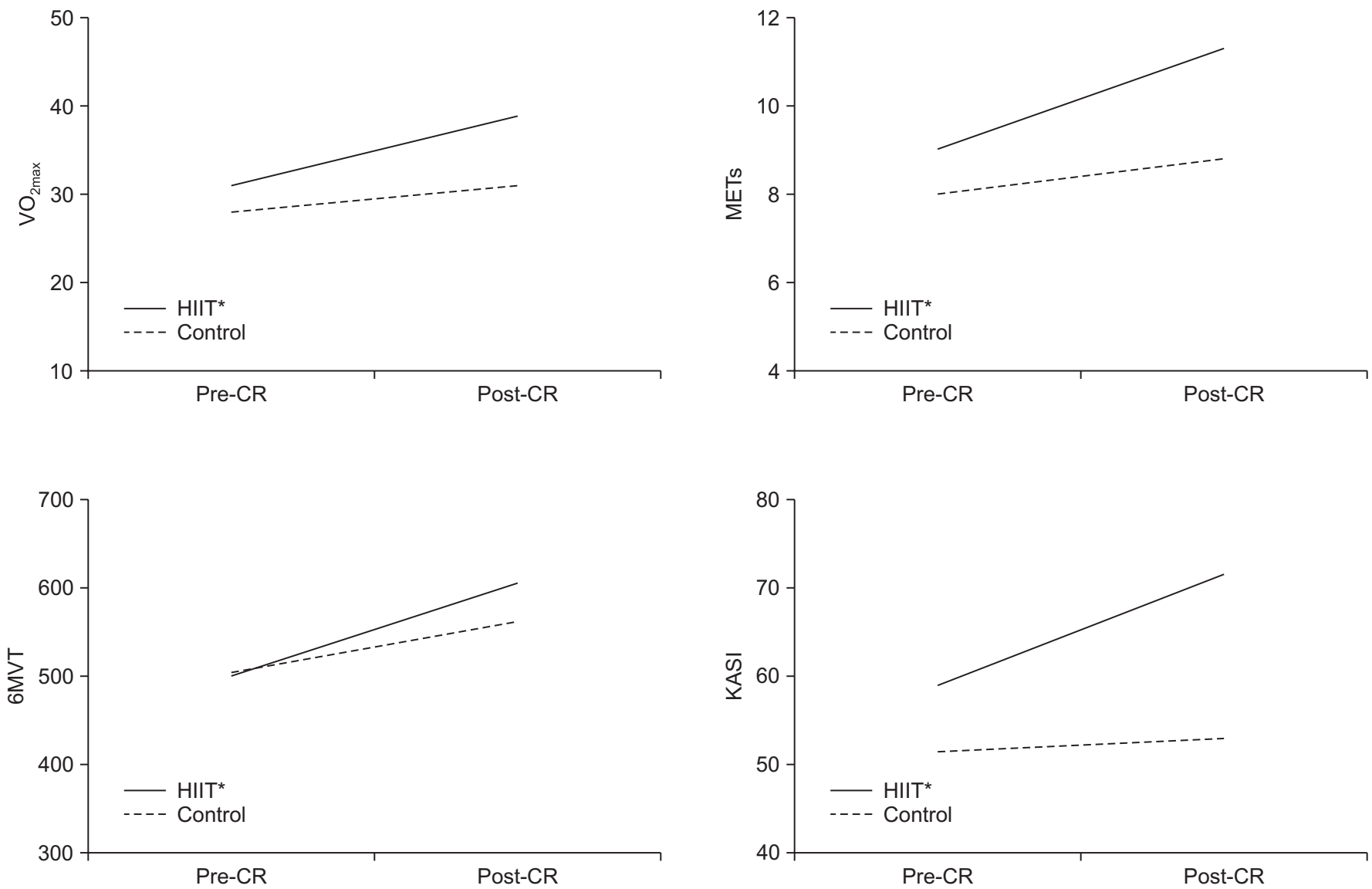

Fig. 3. Improvements in cardiovascular functions in the two study groups $(* \mathrm{p}<0.05)$. CR, cardiac rehabilitation; $\mathrm{VO}_{2 \max }$, maximal oxygen consumption; METs, metabolic equivalents, 6MWT, 6-Minute Walking Test; KASI, Korean Activity Scale/Index. 
$11.67 \pm 3.32$ days in the HIIT group and $17.25 \pm 10.14$ days in the control group. After the first ETT, patients underwent 18 exercise sessions followed by a second ETT. Mean exercise durations were $67.11 \pm 17.53$ days in all patients, $61.47 \pm 13.74$ days in the HIIT group, and $73.41 \pm 30.41$ days in the control group. Time duration from diagnosis to the start of exercise or total exercise duration were not significantly different between HIIT and control groups.

A total of 18 sessions followed an average of 1-2 times a week protocol over an average of 9-10 weeks. Patients who did not complete all 18 sessions due to personal reasons or other morbidity were dropped out. Exercise was performed with sufficient intensity to reach the prescribed HR value. All 44 patients completed 18 sessions of exercise without any adverse event.

Cardiovascular function and activity state were improved after exercise compared to pre-exercise in both HIIT and control group. However, post-exercise cardio-
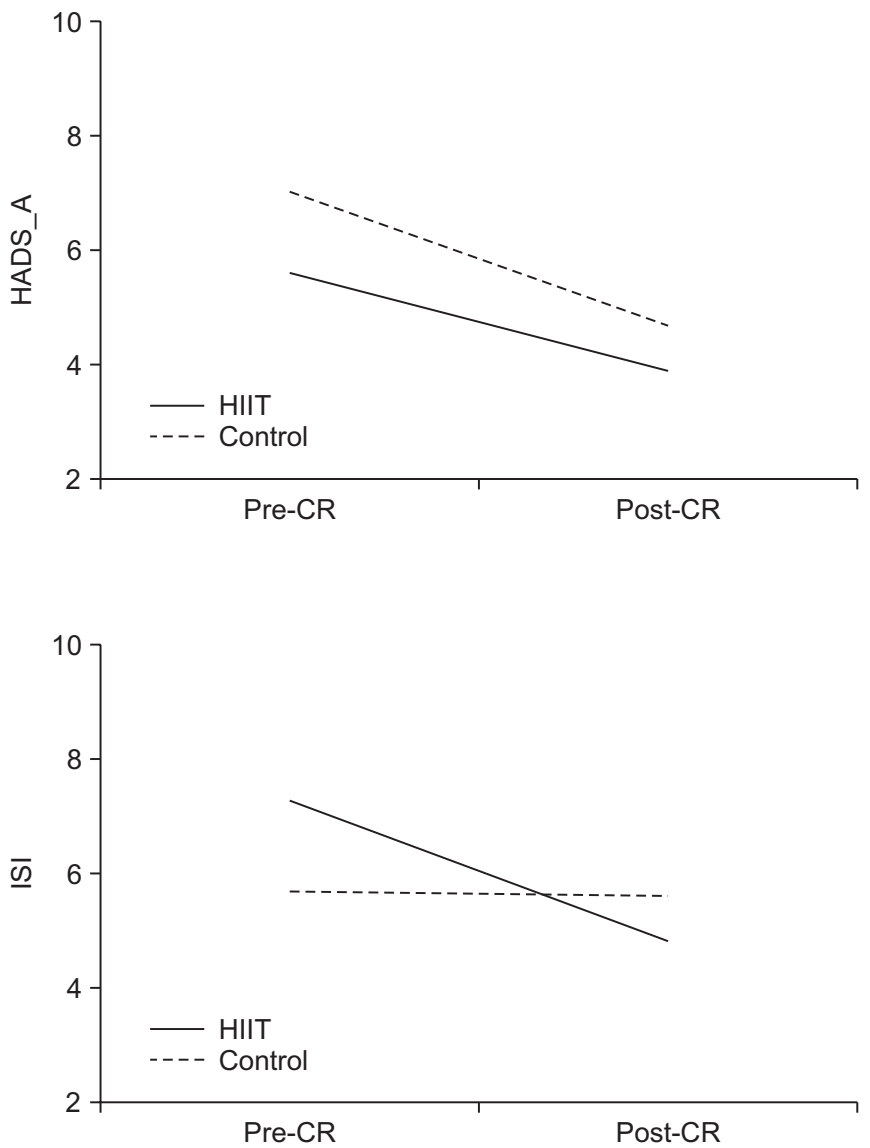

vascular and functional states $\left(\mathrm{VO}_{2 \max }\right.$, METs, $6 \mathrm{MWT}$, and KASI) were improved significantly more in the HIIT group than those in the conventional MICT group (Table 2, Fig. 3). Post-exercise psychological states were also significant different between the two groups (Table 2, Fig. 4). Depression items of HADS (HADS_D) showed significant intergroup differences. Similarly, PHQ-9 (a depression questionnaire) tended to show less depression in the HIIT group ( $\mathrm{p}=0.068$ ) compared to that in the control group. FSS scores (a measure of fatigue) showed greater improvement in the HIIT group than that in the control group. On the other hand, HADS scores for anxiety (HADS_A) and ISI for insomnia were not significantly different between the two groups (Table 2, Fig. 4).

\section{DISCUSSION}

In this study, we examined the effect of HIIT on car-
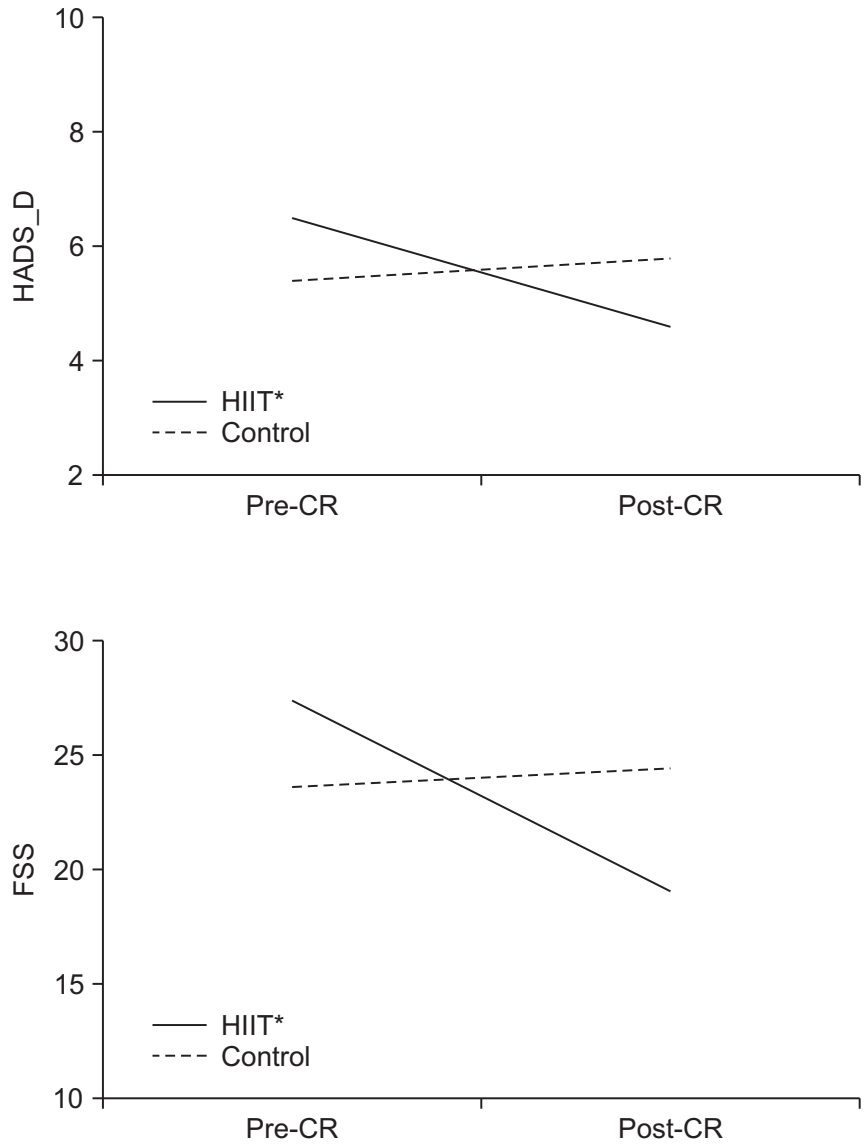

Fig. 4. Improvement of psychological functions in the two study groups $\left({ }^{*} \mathrm{p}<0.05\right)$. CR, cardiac rehabilitation; HADS A, anxiety items of the Hospital Anxiety and Depression Scale; HADS_D, depression items of the Hospital Anxiety and Depression Scale; ISI, Insomnia Severity Index; FSS, Fatigue Severity Scale. 
diovascular functions and psychological symptoms. Our results showed that HIIT was more effective in improving objective cardiovascular functions such as $\mathrm{VO}_{2 \max }$, METs, $6 \mathrm{MWT}$, and subjective functional activity state evaluated by KASI questionnaire than MICT (Table 2, Fig. 3). In addition, HIIT improved psychological symptoms such as depression and fatigue more than MICT.

Increases in $\mathrm{VO}_{2 \max }$ depend on time spent near $\mathrm{VO}_{2 \max }$ during training sessions. HIIT protocols allow patients to exercise at near maximal $\mathrm{VO}_{2 \max }$ for longer durations than conventional MICT in order to improve maximal cardiovascular functions $[6,25]$.

Previous studies have shown that HIIT can achieve better $\mathrm{VO}_{2 \max }$ and METs improvements than MICT. However, the majority of these studies only enrolled low risk patients or did not suggest risk stratification for cardiac rehabilitation. In addition, previous studies on HIIT enrolled patients 3 to 6 months after MI or did not clearly mention about the initial time of CR participation [5-9]. In the present study, we enrolled patients with low and moderate risks that started CR about 14 days after MI. These results could suggest that HIIT is a safe and effective method for improving cardiovascular functions in stable low risk MI and moderate risk MI patients in early phase II session.

We found that KASI scores reflecting patient's subjective activity states were significantly improved in the HIIT group more than those in MICT group (Table 2, Fig. 3). KASI is a Korean version of DASI. It has been used to estimate the likelihood of major adverse cardiac events [26]. Relatively high correlations between KASI scores and $\mathrm{VO}_{2 \max }$ have been reported [19,20]. Although KASI is commonly used to assess functional activity state after MI, the effect of HIIT on KASI scores has not been studied. Results of this study suggest that HIT is a more effective method than MICT for improving objective cardiovascular functions measured by $\mathrm{VO}_{2 \max }$ and subjective activity as well as functional state of MI patients.

Depression has been found in $65 \%$ of MI patients [1417]. Because depressive symptoms are also linked to CR participation and outcome while fatigue can interfere with activities of daily living, participation, and quality of life, optimal methods are needed to improve cardiovascular efficiency and emotional and psychological states of MI patients [27]. In previous studies, the effect of HIIT was mainly assessed in terms of cardiovascular efficacy.
However, in the present study, psychological aspects were also evaluated.

There has been no report on the effect of HIIT on psychological distress. The study by Korzeniowska-Kubacka et al. [28] has shown that 8-week conventional CR can only decrease depression and anxiety in women. However, our results showed that HIIT improved depression whereas MICT did not (Table 2, Fig. 4). Considering that majority of study participants in our study were men (only 5 of 44 participants were women), our results suggest that HIIT could be effective in reducing depression in men with MI. A larger-scale study with longer duration is needed to determine the effect of HIIT on psychological symptoms in males and females.

Fatigue is a general and sustained feeling of exhaustion or difficulty performing physical and mental activities. It negatively affects physical functions, emotional states, activities of daily living, and quality of life $[29,30]$. There has been no previous report about the relationship between fatigue and cardiac rehabilitation including HIIT. Previous studies on HIIT conducted for 4 weeks in healthy people suggested that it was more effective in increasing neuromuscular fatigue threshold than MICT [31]. Similarly, we found that HIIT could more effectively reduce fatigue than MICT (Table 2, Fig. 4). This indicates that HIIT is a better CR method than MICT for addressing fatigue control in MI patients.

In the present study, HIIT did not more effectively reduce anxiety or insomnia than MICT. Although not statistically significant, insomnia was decreased more in the HIIT group compared to that in the control group. A previous study in a general population has shown that depression and anxiety can increase the risk of insomnia and vice versa [32]. However, relationships between depression/anxiety and insomnia are known to be complex. A large scale, long-term study is needed in the future to determine the effect of HIIT on insomnia and anxiety.

This study has some limitations. First, the number of patients recruited was small, although it was enough to meet the calculated required sample size of 20 patients per group. Second, high risk patents were not enrolled in this study because we were not sure of the safety of HIIT for these patients. Third, because our study subjects had returned to society before enrollment, many other factors such as the level of social support might have affected psychological aspects. 
In this study, HIIT was found to be more effective than MICT in improving cardiac functions, activity, and psychological states in low and moderate risk phase II MI patients. This observation suggests that HIIT can more effectively improve cardiac function, activity, and psychological status than MICT. A further larger scale, longer duration study is needed in the future to confirm our results regarding the benefit of HIIT for MI patients.

\section{CONFLICT OF INTEREST}

No potential conflict of interest relevant to this article was reported.

\section{REFERENCES}

1. World Health Organization. The top 10 causes of death (Fact Sheet No. 310). Geneva: World Health Organization; 2017.

2. Bartels MN, Bourne GW, Dwyer JH. High-intensity exercise for patients in cardiac rehabilitation after myocardial infarction. PM R 2010;2:151-5; discussion 155.

3. Lawler PR, Filion KB, Eisenberg MJ. Efficacy of exercise-based cardiac rehabilitation post-myocardial infarction: a systematic review and meta-analysis of randomized controlled trials. Am Heart J 2011;162:57184.

4. Balady GJ, Williams MA, Ades PA, Bittner V, Comoss P, Foody JM, et al. Core components of cardiac rehabilitation/secondary prevention programs: 2007 update. A scientific statement from the American Heart Association Exercise, Cardiac Rehabilitation, and Prevention Committee, the Council on Clinical Cardiology; the Councils on Cardiovascular Nursing, Epidemiology and Prevention, and Nutrition, Physical Activity, and Metabolism; and the American Association of Cardiovascular and Pulmonary Rehabilitation. Circulation 2007;115:2675-82.

5. Rognmo O, Hetland E, Helgerud J, Hoff J, Slordahl SA. High intensity aerobic interval exercise is superior to moderate intensity exercise for increasing aerobic capacity in patients with coronary artery disease. Eur J Cardiovasc Prev Rehabil 2004;11:216-22.

6. Guiraud T, Juneau M, Nigam A, Gayda M, Meyer P, Mekary S, et al. Optimization of high intensity interval exercise in coronary heart disease. Eur J Appl Physiol
2010;108:733-40.

7. Fletcher GF, Balady GJ, Amsterdam EA, Chaitman B, Eckel R, Fleg J, et al. Exercise standards for testing and training: a statement for healthcare professionals from the American Heart Association. Circulation 2001;104:1694-740.

8. Warburton DE, McKenzie DC, Haykowsky MJ, Taylor A, Shoemaker P, Ignaszewski AP, et al. Effectiveness of high-intensity interval training for the rehabilitation of patients with coronary artery disease. Am J Cardiol 2005;95:1080-4.

9. Munk PS, Staal EM, Butt N, Isaksen K, Larsen AI. High-intensity interval training may reduce in-stent restenosis following percutaneous coronary intervention with stent implantation: a randomized controlled trial evaluating the relationship to endothelial function and inflammation. Am Heart J 2009;158:734-41.

10. Lichtman JH, Bigger JT Jr, Blumenthal JA, FrasureSmith N, Kaufmann PG, Lesperance F, et al. Depression and coronary heart disease: recommendations for screening, referral, and treatment. A science advisory from the American Heart Association Prevention Committee of the Council on Cardiovascular Nursing, Council on Clinical Cardiology, Council on Epidemiology and Prevention, and Interdisciplinary Council on Quality of Care and Outcomes Research: endorsed by the American Psychiatric Association. Circulation 2008;118:1768-75.

11. Kooistra M, van der Graaf Y, Grool AM, Zuithoff NP, Jan Biessels G, Geerlings MI, et al. The natural course of elevated levels of depressive symptoms in patients with vascular disease over eight years of follow-up. The SMART-Medea study. J Affect Disord 2016;202:95101.

12. Huffman JC, Celano CM, Beach SR, Motiwala SR, Januzzi JL. Depression and cardiac disease: epidemiology, mechanisms, and diagnosis. Cardiovasc Psychiatry Neurol 2013;2013:695925.

13. O'connor CM, Gurbel PA, Serebruany VL. Depression and ischemic heart disease. Am Heart J 2000;140(4 Suppl):63-9.

14. Wenger NK. Current status of cardiac rehabilitation. J Am Coll Cardiol 2008;51:1619-31.

15. Yohannes AM, Doherty P, Bundy C, Yalfani A. The long-term benefits of cardiac rehabilitation on depression, anxiety, physical activity and quality of life. J 
Clin Nurs 2010;19:2806-13.

16. Milani RV, Lavie CJ. Impact of cardiac rehabilitation on depression and its associated mortality. Am J Med 2007;120:799-806.

17. Duarte Freitas P, Haida A, Bousquet M, Richard L, Mauriege P, Guiraud T. Short-term impact of a 4-week intensive cardiac rehabilitation program on quality of life and anxiety-depression. Ann Phys Rehabil Med 2011;54:132-43.

18. Moholdt T, Madssen E, Rognmo O, Aamot IL. The higher the better? Interval training intensity in coronary heart disease. J Sci Med Sport 2014;17:506-10.

19. Sung J, On YK, Kim HS, Chae IH, Sohn DW, Oh BH, et al. Development of Korean Activity Scale/Index (KASI). Korean Circ J 2000;30:1004-9.

20. Hlatky MA, Boineau RE, Higginbotham MB, Lee KL, Mark DB, Califf RM, et al. A brief self-administered questionnaire to determine functional capacity (the Duke Activity Status Index). Am J Cardiol 1989;64:6514.

21. Zigmond AS, Snaith RP. The hospital anxiety and depression scale. Acta Psychiatr Scand 1983;67:361-70.

22. Kroenke K, Spitzer RL, Williams JB. The PHQ-9: validity of a brief depression severity measure. J Gen Intern Med 2001;16:606-13.

23. Valko PO, Bassetti CL, Bloch KE, Held U, Baumann CR. Validation of the fatigue severity scale in a Swiss cohort. Sleep 2008;31:1601-7.

24. Bastien $\mathrm{CH}$, Vallieres A, Morin CM. Validation of the Insomnia Severity Index as an outcome measure for insomnia research. Sleep Med 2001;2:297-307.

25. Thevenet D, Tardieu M, Zouhal H, Jacob C, Abderrahman BA, Prioux J. Influence of exercise intensity on time spent at high percentage of maximal oxygen uptake during an intermittent session in young endurance-trained athletes. Eur J Appl Physiol 2007;102:19-
26.

26. Shaw LJ, Olson MB, Kip K, Kelsey SF, Johnson BD, Mark DB, et al. The value of estimated functional capacity in estimating outcome: results from the NHBLIsponsored Women's Ischemia Syndrome Evaluation (WISE) Study. J Am Coll Cardiol 2006;47(3 Suppl): S36-43.

27. Johnston M, Foulkes J, Johnston DW, Pollard B, Gudmundsdottir $\mathrm{H}$. Impact on patients and partners of inpatient and extended cardiac counseling and rehabilitation: a controlled trial. Psychosom Med 1999;61:22533.

28. Korzeniowska-Kubacka I, Bilinska M, Piotrowska D, Stepnowska M, Piotrowicz R. The impact of exerciseonly-based rehabilitation on depression and anxiety in patients after myocardial infarction. Eur J Cardiovasc Nurs 2017;16:390-6.

29. Evangelista LS, Moser DK, Westlake C, Pike N, TerGalstanyan A, Dracup K. Correlates of fatigue in patients with heart failure. Prog Cardiovasc Nurs 2008; 23:12-7.

30. Kim YJ, Rogers JC, Raina KD, Callaway CW, Rittenberger JC, Leibold ML, et al. An intervention for cardiac arrest survivors with chronic fatigue: a feasibility study with preliminary outcomes. Resuscitation 2016;105:109-15.

31. Miramonti AA, Stout JR, Fukuda DH, Robinson EH 4th, Wang R, La Monica MB, et al. Effects of 4 weeks of high-intensity interval training and $\beta$-hydroxy- $\beta$ methylbutyric free acid supplementation on the onset of neuromuscular fatigue. J Strength Cond Res 2016; 30:626-34.

32. Roberts RE, Shema SJ, Kaplan GA, Strawbridge WJ. Sleep complaints and depression in an aging cohort: a prospective perspective. Am J Psychiatry 2000;157:81-8. 